\title{
Remote pharmacological post-conditioning by intrathecal morphine: cardiac protection from spinal opioid receptor activation
}

\author{
J. Ling Ling, G. T. C. Wong, L. YAO, Z. XIA and M. G. IrwiŇ \\ Department of Anaesthesiology, University of Hong Kong, Hong Kong
}

\begin{abstract}
Background: Intrathecal morphine pre-conditioning attenuates cardiac ischemia-reperfusion injury via activation of central opioid receptors. We hypothesized that intrathecal morphine also post-conditions the myocardium in the rat.

Methods: Intrathecal morphine at $0.3 \mu \mathrm{g} / \mathrm{kg}$ (LMPC), $3 \mu \mathrm{g} / \mathrm{kg}$ (MMPC) or $30 \mu \mathrm{g} / \mathrm{kg}$ (HMPC) was administered for $5 \mathrm{~min}$ before 120 -min reperfusion following 30-min ischemia. Infarct size as a percentage of area at risk (IS / AAR) was determined using triphenyltetrazolium staining. MMPC was repeated following the intrathecal administration of nor BNI, NTD, CTOP, or naloxone methiodide (NM), kappa, delta, mu and non-specific opioid receptor antagonists, respectively. The role of peripheral opioid, adenosine and calcitonin gene-related peptide (CGRP) receptors was examined by the intravenous administration of NM, 8- $\rho$-sulfophenyl theophylline (8-SPT) and human CGRP fragment $\left(\mathrm{CGRP}_{8-37}\right)$, respectively.
\end{abstract}

Results: Morphine post-conditioning at all three doses was cardioprotective (IS/AAR of LMPC $=37 \pm 4 \%, \mathrm{MMPC}=35 \pm 5 \%$, $\mathrm{HMPC}=32 \pm 4 \%$, control $=50 \pm 5 \%, P<0.01)$. The prior administration of opioid receptor antagonists intrathecally, as well as intravenous 8-SPT and CGRP ${ }_{8-37}$ receptor antagonists, abolished this effect (nor BNI+MMPC $=47 \pm 7 \%$, NTD $+\mathrm{MMPC}=49 \pm 7 \%$, $\mathrm{CTOP}+\mathrm{MMPC}=45 \pm 9 \%, \quad \mathrm{NM}+\mathrm{MMPC}=47 \pm 6 \% \quad 8$-SPT + $\left.\mathrm{MPC}=46 \pm 5 \% \& \mathrm{CGRP}_{8-37}+\mathrm{MPC}=53 \pm 6 \%, P=0.63\right)$. However, the intravenous administration of $\mathrm{NM}$ did not prevent the protective effect $(34 \pm 4 \%, P<0.01)$.

Conclusions: Intrathecal morphine administration can induce pharmacological cardiac post-conditioning as it involves opioid receptor centrally but non-opioid receptors peripherally.

Accepted for publication 21 July 2010

(C) 2010 The Authors

Journal compilation (C) 2010 The Acta Anaesthesiologica Scandinavica Foundation
$\mathrm{R}$ ESTORATION of blood flow following a period of ischemia is known to induce cellular damage in addition to that caused by the ischemia itself. ${ }^{1,2}$ Intervention at the time of reperfusion (post-conditioning) has been clearly demonstrated to ameliorate ischemia-reperfusion (IR) injury and can be achieved by intermittent IR of the area sustaining the ischemic insult, as well as by treatment with a number of drugs. ${ }^{3}$ Ischemic post-conditioning of another organ or even to the non-ischemic areas within the same organ can also reduce IR injury (remote post-conditioning). ${ }^{4}$ It is difficult, however, to ascertain whether pharmacological post-conditioning is an entirely local or remote phenomenon as both the target and the remote organs are exposed to the conditioning agent.

Cardiac opioid receptors are known to be involved in both ischemic and opioid post-conditioning. ${ }^{5-7}$ Clinically available opioids such as morphine ${ }^{7,8}$ and remifentanil ${ }^{9,10}$ have been shown to be cardioprotective when administered intravenously in both a pre-conditioning and a post-conditioning manner. Administration of intrathecal morphine before the index ischemic event can attenuate cardiac IR injury to the same extent as intravenous morphine preconditioning. ${ }^{11}$ However, this protective effect involves the activation of central but not peripheral opioid receptors, ${ }^{12}$ pointing to the involvement of non-opioid myocardial receptors in mediating this process. The adenosine receptor was one of the first G-protein-coupled receptors to be linked to the ischemic post-conditioning process ${ }^{13}$ and adenosine is well known to be involved with cardiac protection. The neuropeptide calcitonin gene-related peptide (CGRP) is major neurotransmitter in sensory C fibers ${ }^{14}$ and have been shown to be involved in both pre-conditioning and post-conditioning of myocardial tissue. ${ }^{15,16}$ Both of these receptor types are

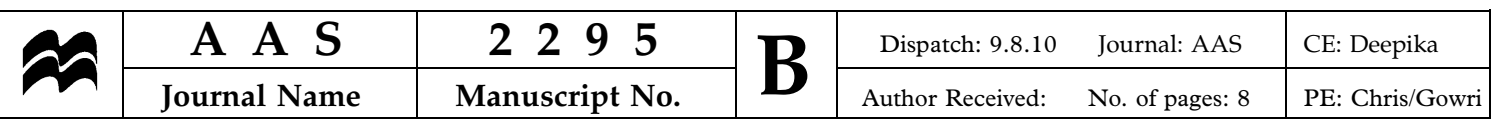




\section{J. Ling Ling et al.}

widely distributed in cardiovascular tissues. It is possible that some of these receptors may be remotely activated from intrathecal morphine administration. As central opioid receptor agonist activity can remotely pre-condition the myocardium, we hypothesized that intrathecal morphine post-conditioning can also protect the myocardium against IR injury through peripheral non-opioid receptors. We also investigated the possible involvement of the adenosine and CGRP receptors in this process.

\section{Methods}

Approval was obtained from our institutional Committee for the Use of Live Animals in Teaching and Research before the commencement of all experimental procedures. A total of 140, 8-weekold Sprague-Dawley rats, weighing $300 \pm 25 \mathrm{~g}$, were used for the study. The animals were housed in separate cages, exposed to 12-h light/dark cycles and given free access to food and water.

\section{Intrathecal catheter placement}

Following induction of anesthesia using intraperitoneal pentobarbitone $(50 \mathrm{mg} / \mathrm{kg})$ and skin preparation with $70 \%$ alcohol, a polyethylene-10 catheter (external diameter $0.8 \mathrm{~mm}$ and internal diameter $0.4 \mathrm{~mm}$ ) was inserted through a puncture in the atlanto-occipital membrane. The catheter was then advanced $4 \mathrm{~cm}$ to the thoracic spinal cord level according to the method of Yaksh and Rudy, $^{17}$ with cerebrospinal fluid (CSF) backflow through the lumen indicating successful placement. After wound closure, the animals were returned to their cages for recovery and observed for the next 3 days. Animals displaying signs of gross motor or sensory deficits were excluded from further study. As an additional confirmation of appropriate catheter placement, Evans blue dye was injected through the intrathecal catheter at necropsy and the spinal cord was inspected for macroscopic evidence of damage.

\section{IR injury}

After a minimum of 3 days post-intrathecal catheter placement, an open-chest model was used to produce IR injury. Anesthesia was induced using intraperitoneal pentobarbitone $(50 \mathrm{mg} / \mathrm{kg})$ and supplemented $(25 \mathrm{mg} / \mathrm{kg})$ if there were signs of light anesthesia indicated by movement of the animal. The lungs were mechanically ventilated with a Harvard Apparatus Rodent Respirator (Holliston, MA) with room air at $60-70$ breaths $/ \mathrm{min}$ through a tracheostomy tube. Body temperature was monitored and maintained at $37 \pm 1^{\circ} \mathrm{C}$ using a heating pad. The carotid artery was cannulated with a polyethylene catheter for direct arterial blood pressure monitoring and the right jugular vein was similarly cannulated for drug or saline infusion. Electrocardiogram (lead II) monitoring was achieved using subcutaneous stainless-steel electrodes that were connected via a cable to a PowerLab monitoring system (ML750 PowerLab/ 4sp with MLT0380 Reusable BP Transducer; AD Instruments, CO Springs, $\mathrm{CO}$ ).

Once all monitoring and vascular access were established, the heart was exposed via thoracotomy at the left fifth intercostal space, the pericardium was removed and a 6-0 loop of suture was placed with a snare occluder encircling the origin of the left main coronary artery. Regional myocardial ischemia was then induced by tightening the snare and securing the threads with a hemostat, seeking confirmatory signs of cyanosis in the distribution area of the left coronary artery, ST segment changes in the electrocardiogram, and a decrease in mean arterial blood pressure (MAP). Animals developing intractable ventricular fibrillation or severe hypotension (MAP $<30 \mathrm{mmHg}$ ) during the ischemic period were excluded from further study without proceeding to post-conditioning treatments. Toward the end of the ischemic period, different post-conditioning maneuvers were performed. The animals underwent a total of $30 \mathrm{~min}$ of ischemia followed by $120 \mathrm{~min}$ of reperfusion.

\section{Infarct size determination}

The hearts were excised and transferred to a Langendorff apparatus at the end of the reperfusion period. The infarct size (IS) was determined as a percentage of the area placed at risk (AAR), using a combination of triphenyltetrazolium and Evan Blue staining, and then quantification was performed using a computerized planimetry technique. The IS/AAR ratio was used to compare the differences between groups.

\section{Study groups and experimental protocol}

Two series of experiments were performed concurrently, with the same control group being used for both series. The first series determined the presence of an anti-infarct effect with intrathecal morphine 
post-conditioning and compared it with that of ischemic and intravenous morphine post-conditioning. The second series examined the effects that prior administration of opioid, adenosine and CGRP receptor antagonists had on intrathecal morphine post-conditioning. Treatment order followed a predetermined randomized sequence generated for each series. Intrathecal morphine post-conditioning (MPC) was induced by a 5-min infusion of morphine just before reperfusion at doses of $0.3,3$ or $30 \mu \mathrm{g} / \mathrm{kg}$ (LMPC, MMPC and HMPC, respectively). Positive controls comprised of intravenous morphine and ischemic post-conditioning, while the negative control group received intrathecal normal saline. Intravenous morphine post-conditioning (IVMPC) was achieved using $300 \mu \mathrm{g} / \mathrm{kg}$ of intravenous morphine and ischemic post-conditioning (IPOC) consisted of three cycles of 30-s reperfusion and 30-s ischemia. In the second series of experiments, $15 \mathrm{nmol}$ of the selective kappa, delta and $\mathrm{mu}$ OR antagonists were administered intrathecally $10 \mathrm{~min}$ before MPC $(3 \mu \mathrm{g} / \mathrm{kg})$. The compounds were nor-binaltorphimine (nor BNI), naltrindole (NTD) and D-Phe-Cys-Tyr-D-Trp-OrnThr-Pen-Thr-NH $\mathrm{N}_{2}$ (CTOP), respectively (Sigma Chemical Company, St Louis, MO). The quaternary non-specific opioid antagonist naloxone methiodide $(20 \mu \mathrm{g} / \mathrm{kg})$ was administered either intrathecally (NM) or intravenously (IVNM). The nonselective adenosine receptor antagonist 8 - $\rho$-sulfophenyl theophylline (8-SPT) was administered intravenously at a dose of $7.5 \mathrm{mg} / \mathrm{kg}^{18}$ and the CGRP receptor antagonist hCGRP ${ }_{8-37}$ was administered at a dose of $0.01 \mathrm{nmol} / \mathrm{kg} .{ }^{19}$

Each of these compounds was administered, respectively, in the absence of intrathecal morphine to evaluate any infarct-sparing effects. cals were dissolved in normal saline and tered in a volume of $10 \mu \mathrm{l}$ (Fig. 1).

\section{Statistical analysis}

Using this same model of cardiac IR injury, data from previous experiments indicated the expected IS/AAR of the control group to be between $50 \%$ and $65 \%$ and the magnitude of IS/AAR reduction to be at least $50 \%$. Therefore, five animals per group would be required to yield a power of $80 \%$ and a $P$ value of 0.05 . All data were expressed as mean $\pm S D$ and were obtained from six separate animals per group. For infarct size data, one-way analysis of variance was performed with StudentNewman-Keul tests for multiple comparisons.

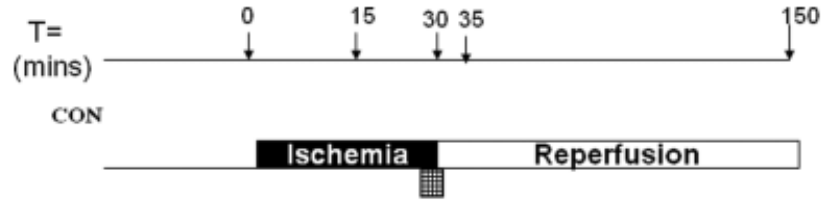

IPoC

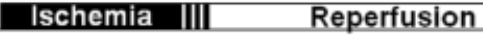

Morphine

Postconditioning
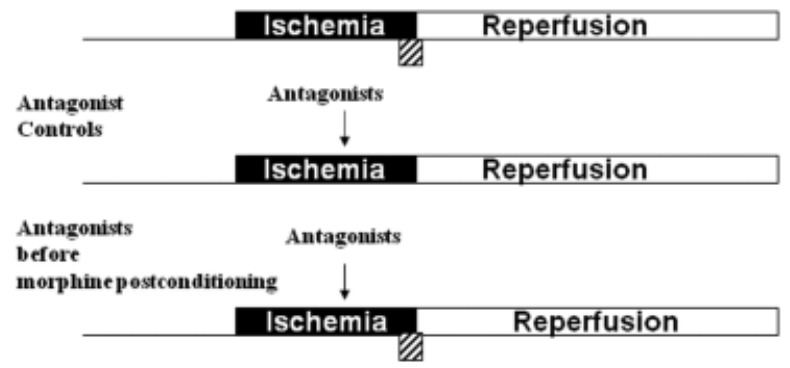

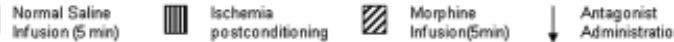

Fig. 1. Bar graph depicting the experimental protocols. Morphine post-conditioning consisted of one of the following regimes: $300 \mu \mathrm{g} / \mathrm{kg}$ intravenous morphine; $0.3,3$ or $30 \mu \mathrm{g} / \mathrm{kg}$ intrathecal morphine. Antagonists consisted of one the following: intrathecal nor BNI, CTOP, NTD or naloxone methiodide; intravenous naloxone methiodide, $8-S P T$ or $C G R P_{8-37}$. The antagonists were individually administered either alone or before morphine post-conditioning $(3 \mu \mathrm{g} / \mathrm{kg})$. CON, control; IPoC, ischemic postconditioining.

Hemodynamic data were analyzed using ANOVA for between-group comparisons, with the Bonferroni correction applied for multiple comparisons if significant $F$ ratios were obtained. Repeated-measure analysis of variance was used to compare between time points within each group. Statistical analysis was performed using a personal computer software program (SPSS version 16.0 for Windows, Chicago, IL) and a $P$ value of $<0.05$ was considered significant.

\section{Results}

A total of 140 animals were used. Six rats suffered neurological damage following intrathecal catheter placement and 14 experienced severe hypotension or ventricular fibrillation and were excluded from further study. These events occurred before administration of any chemicals and therefore not included in the randomized process. The hemodynamic data were comparable at baseline between the groups and are presented in Tables 1 and 2 . Ischemic post-conditioning significantly reduced 
Table 1

Hemodynamic parameters for the first series of experiments.

\begin{tabular}{lccccc}
\hline Group & $n$ & Baseline & Ischemia & Treatment & Reperfusion \\
\hline HR & & & & & \\
Control & 6 & $351 \pm 59$ & $351 \pm 66$ & $333 \pm 67$ & $330 \pm 62$ \\
LMPC & 6 & $369 \pm 61$ & $364 \pm 35$ & $327 \pm 62 \dagger$ & $338 \pm 33$ \\
MMPC & 6 & $361 \pm 39$ & $343 \pm 53$ & $307 \pm 39 \dagger$ & $322 \pm 52$ \\
HMPC & 6 & $387 \pm 26$ & $348 \pm 35 \dagger$ & $341 \pm 52 \dagger$ & $286 \pm 52 \dagger$ \\
IVMPC & 6 & $401 \pm 31$ & $350 \pm 44 \dagger$ & $306 \pm 45 \dagger$ & $324 \pm 40 \dagger$ \\
POC & 6 & $369 \pm 69$ & $333 \pm 48 \dagger$ & $323 \pm 60 \dagger$ & $260 \pm 34 \dagger$ \\
MAP & & & & & \\
Control & 6 & $105 \pm 24$ & $76 \pm 22 \dagger$ & $77 \pm 19 \dagger$ & $93 \pm 25$ \\
LMPC & 6 & $113 \pm 15$ & $71 \pm 14 \dagger$ & $79 \pm 15 \dagger$ & $89 \pm 8 \dagger$ \\
MMPC & 6 & $101 \pm 9$ & $68 \pm 14 \dagger$ & $74 \pm 7 \dagger$ & $88 \pm 8$ \\
HMPC & 6 & $111 \pm 10$ & $75 \pm 16 \dagger$ & $87 \pm 18 \dagger$ & $82 \pm 13 \dagger$ \\
IVMPC & 6 & $93 \pm 23$ & $61 \pm 12 \dagger$ & $61 \pm 14 \dagger$ & $77 \pm 25 \dagger$ \\
IPOC & 6 & $107 \pm 12$ & $63 \pm 12 \dagger$ & $55 \pm 10^{\star}, \dagger$ & $83 \pm 16 \dagger$ \\
\hline
\end{tabular}

Values were obtained at the end of the respective periods.

${ }^{*} P<0.05$ compared with control (between group comparison, one-way ANOVA).

$\dagger P<0.05$ compared with baseline (within group comparison, repeated measure ANOVA).

$\mathrm{HR}$, heart rate (beats per min); MAP, mean arterial pressure $(\mathrm{mmHg})$; IPOC, ischemic post-conditioning; IVMPC, intravenous morphine post-conditioning; LMPC, low dose intrathecal morphine post-conditioning $(0.3 \mu \mathrm{g} / \mathrm{kg})$; MMPC, medium dose intrathecal morphine post-conditioning $(3 \mu \mathrm{g} / \mathrm{kg})$; HMPC, highdose intrathecal morphine post-conditioning $(30 \mu \mathrm{g} / \mathrm{kg})$.

the mean arterial pressure (MAP) during the treatment period compared with control.

Intrathecal morphine at all three doses reduced the infarct size compared with the control group $(\mathrm{LMPC}=37 \pm 4 \%, \quad \mathrm{MMPC}=35 \pm 5 \%, \mathrm{HMPC}=32$ $\pm 4 \%$, control $=50 \pm 5 \%, P<0.01)$. There was no difference when the MMPC and HMPC were compared with ischemic $(30 \pm 6 \%)$ and intravenous morphine post-conditioning $(29 \pm 5 \%) \quad(P=0.13$, Fig. 2). The sole administration of any one of the antagonists did not change the infarct size compared with the control group (Fig. 3$)(P=0.81)$. The prior intrathecal administration of any one of the opioid receptor antagonists as well as the intravenous administration of both 8-SPT and $\mathrm{CGRP}_{8-37}$ abolished the protective effect of morphine post-conditioning (nor $\mathrm{BNI}+\mathrm{MPC}=47 \pm 7 \%, \mathrm{CTOP}+\mathrm{MPC}=$ $45 \pm 9 \%, \quad \mathrm{NTD}+\mathrm{MPC}=49 \pm 7 \%, \quad \mathrm{NM}+\mathrm{MPC}=$ $47 \pm 6 \%, 8-\mathrm{SPT}+\mathrm{MPC} 46 \pm 5 \%$ and $\mathrm{CGRP}_{8-37}+$ MPC $53 \pm 6 \%, P=0.63)$. However, the intravenous administration of NM did not abolish the protective effect $(34 \pm 4 \%, P<0.01)$ (Fig. 4$)$.

\section{Discussion}

Our results show that intrathecal morphine can reduce myocardial infarct size at a fraction of the intravenous dose when administered after the start of an index ischemic event and just before reperfusion. Interestingly, this occurs at a much lower dose than what is required to produce this effect intravenously. Agonism of any one of the three opioid receptor subtypes at the spinal cord can activate the cardioprotective process, as blockade of any one of them is sufficient to attenuate this response. In contrast, peripheral opioid receptors are not involved in this process as intravenous naloxone methiodide, a peripherally restricted non-selective opioid antagonist, failed to block the cardioprotective effect. Rather, other receptors such as adenosine and CGRP receptors are involved as their respective antagonists are able to suppress the cardioprotective response. This pattern of cardiac protection is similar to that achieved with intrathecal morphine pre-conditioning. ${ }^{11}$ Given the slow and limited ability of intrathecal morphine to reach the systemic circulation, ${ }^{20}$ the short therapeutic window within which systemic opioid post-conditioning is effective ${ }^{21}$ and the lack of peripheral opioid receptor involvement, it is unlikely that morphine itself is the peripheral trigger. Thus, this is a form of remote pharmacological postconditioning, with the central nervous system and the heart being the remote and the target organs, respectively. As the receptor types for the remote and the target organs differ, this mode of triggering remote post-conditioning of the heart most likely involves secondary signaling mechanisms.

Although innately powerful, the application of ischemic pre-conditioning to clinical practice is limited by the need to intervene before the ischemic event with an invasive protocol. The concept of remote ischemic post-conditioning, first reported by Kerendi et al., ${ }^{22}$ can conveniently overcome both these obstacles. Post-conditioning can also be mimicked by a host of pharmacological agents, including drugs commonly used in the perioperative period such as inhalational anesthetics ${ }^{23,24}$ and

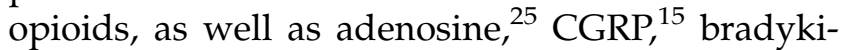
nin $^{26}$ and erthryopoietin. ${ }^{27}$ As both the target and the remote organs may simultaneously be exposed to the agents, the demarcation between remote and local pharmacological post-conditioning is less distinct, though arguably less important. Classical ischemic pre- and post-conditioning have been shown to share some common pathways. ${ }^{28}$ It is, therefore, not inconceivable that remote pre- and post-conditioning may similarly share certain characteristics. As for remote ischemic pre-conditioning, existing evidence indicates the possible 
Table 2

\begin{tabular}{|c|c|c|c|c|c|}
\hline Group & $n$ & Baseline & Ischemia & Treatment & Reperfusion \\
\hline \multicolumn{6}{|l|}{ HR } \\
\hline Control & 6 & $351 \pm 59$ & $351 \pm 66$ & $333 \pm 67$ & $330 \pm 62$ \\
\hline Nor-BNI & 6 & $356 \pm 64$ & $283 \pm 55^{\star}$ & $302 \pm 74^{\star}$ & $297 \pm 56^{*}$ \\
\hline CTOP & 6 & $396 \pm 41$ & $357 \pm 34^{*}$ & $330 \pm 47^{\star}$ & $312 \pm 53^{*}$ \\
\hline NTD & 6 & $394 \pm 67$ & $354 \pm 54^{*}$ & $311 \pm 74^{*}$ & $319 \pm 80^{*}$ \\
\hline NM & 6 & $376 \pm 41$ & $353 \pm 36$ & $360 \pm 33$ & $337 \pm 40$ \\
\hline IVNM & 6 & $407 \pm 26$ & $357 \pm 20$ & $340 \pm 38$ & $330 \pm 44^{*}$ \\
\hline IV 8-SPT & 6 & $392 \pm 13$ & $333 \pm 18^{*}$ & $321 \pm 18^{\star}$ & $325 \pm 30^{*}$ \\
\hline IV CGRP $_{8-37}$ & 6 & $381 \pm 28$ & $325 \pm 27^{\star}$ & $332 \pm 43^{\star}$ & $333 \pm 35$ \\
\hline Nor-BNI+MPC & 6 & $375 \pm 63$ & $387 \pm 54$ & $365 \pm 52$ & $333 \pm 40^{*}$ \\
\hline $\mathrm{CTOP}+\mathrm{MPC}$ & 6 & $372 \pm 31$ & $317 \pm 38^{*}$ & $308 \pm 53^{\star}$ & $299 \pm 43^{*}$ \\
\hline NTD+MPC & 6 & $367 \pm 61$ & $371 \pm 45$ & $375 \pm 22$ & $352 \pm 43$ \\
\hline $\mathrm{NM}+\mathrm{MPC}$ & 6 & $394 \pm 49$ & $396 \pm 44$ & $326 \pm 40^{*}$ & $324 \pm 57^{\star}$ \\
\hline IVNM+MPC & 6 & $423 \pm 16$ & $389 \pm 22$ & $354 \pm 28^{*}$ & $328 \pm 21^{*}$ \\
\hline IV 8-SPT+MPC & 6 & $390 \pm 33$ & $356 \pm 20$ & $348 \pm 14$ & $354 \pm 26$ \\
\hline IV CGRP $8-37+$ MPC & 6 & $387 \pm 28$ & $354 \pm 11$ & $340 \pm 19$ & $343 \pm 28$ \\
\hline \multicolumn{6}{|l|}{ MAP } \\
\hline Control & 6 & $105 \pm 24$ & $76 \pm 22^{*}$ & $77 \pm 19^{\star}$ & $93 \pm 25$ \\
\hline Nor-BNI & 6 & $100 \pm 23$ & $57 \pm 15^{\star}$ & $57 \pm 14^{*}$ & $77 \pm 19^{*}$ \\
\hline CTOP & 6 & $107 \pm 25$ & $67 \pm 14^{*}$ & $67 \pm 9^{*}$ & $78 \pm 14^{*}$ \\
\hline NTD & 6 & $103 \pm 27$ & $70 \pm 17^{*}$ & $67 \pm 15^{\star}$ & $81 \pm 28^{*}$ \\
\hline NM & 6 & $103 \pm 9$ & $80 \pm 11^{*}$ & $82 \pm 9^{*}$ & $83 \pm 13^{*}$ \\
\hline IVNM & 6 & $111 \pm 18$ & $83 \pm 11^{*}$ & $82 \pm 19^{*}$ & $91 \pm 12$ \\
\hline IV 8-SPT & 6 & $95 \pm 10$ & $88 \pm 4$ & $69 \pm 5^{\star}$ & $79 \pm 8$ \\
\hline IV CGRP $_{8-37}$ & 6 & $93 \pm 16$ & $76 \pm 12$ & $64 \pm 9$ & $73 \pm 17$ \\
\hline Nor-BNI+MPC & 6 & $106 \pm 21$ & $75 \pm 19^{\star}$ & $80 \pm 21^{*}$ & $86 \pm 13^{*}$ \\
\hline CTOP+MPC & 6 & $104 \pm 18$ & $64 \pm 14^{\star}$ & $69 \pm 18^{\star}$ & $71 \pm 16^{*}$ \\
\hline NTD+MPC & 6 & $102 \pm 26$ & $68 \pm 11^{*}$ & $73 \pm 11^{*}$ & $76 \pm 18^{*}$ \\
\hline $\mathrm{NM}+\mathrm{MPC}$ & 6 & $111 \pm 26$ & $83 \pm 29^{*}$ & $81 \pm 22^{*}$ & $95 \pm 26$ \\
\hline IVNM+MPC & 6 & $108 \pm 17$ & $72 \pm 17^{*}$ & $76 \pm 13^{\star}$ & $101 \pm 14$ \\
\hline IV 8-SPT+MPC & 6 & $110 \pm 18$ & $82 \pm 14^{*}$ & $80 \pm 11^{*}$ & $88 \pm 10^{*}$ \\
\hline IV CGRP $8-37+$ MPC & 6 & $109 \pm 15$ & $69 \pm 10^{*}$ & $82 \pm 9^{*}$ & $91 \pm 9$ \\
\hline
\end{tabular}

Values were obtained at the end of the respective periods.

${ }^{\star} P<0.05$ compared with baseline (within group comparison, repeated measure ANOVA).

$\mathrm{HR}$, heart rate (beats per min); MAP, mean arterial pressure $(\mathrm{mmHg})$; MPC, morphine post-conditioning at $3 \mu \mathrm{g} / \mathrm{kg}$; nor $\mathrm{BNI}$, norbinaltorphimine; CTOP, D-Phe-Cys-Tyr-D-Trp-Orn-Thr-Pen-Thr-NH2; NTD, naltrindole; NM, naloxone methiodide; IVNM, intravenous naloxone methiodide; 8-SPT, 8- $\rho$-sulfophenyl theophylline; $\mathrm{CGRP}_{8-37}$, human fragment 8-37 of calcitonin gene-related peptide.

involvement of neural, humoral and inflammatory mechanisms in the transfer of protection from the remote to the target organ. Although the mechanisms underlying this transfer of protection with remote post-conditioning are less clear, recent studies suggest characteristics similar to that of its preconditioning counterpart. ${ }^{29}$

A popular theory for remote ischemic pre-conditioning involves the release of humoral substances from the remote organ, which is then transmitted in the blood to the target organ. This concept is supported by the observation that plasma from one preconditioned animal affords protective properties when transferred to another, independent of neurogenic pathways and even across species. ${ }^{30}$ This presupposes circulatory access by the putative protective substance to the target tissue at the time of reperfusion. A similar concept has been proposed in the case of remote post-conditioning where adenosine has been implicated as one of the circulating mediators. ${ }^{22}$ The strength of post-conditioning protection appears to be dependent on the volume of tissue exposed to ischemia and the duration of the ischemic stimulus applied to the remote organ. ${ }^{31}$ This observation lends itself to the consideration that a threshold needs to be crossed with the remote ischemic post-conditioning stimulus in order for it to confer cardioprotective properties, perhaps with the threshold correlating with the amount of circulating factors generated and released. Intrathecal morphine itself is not known to generate ischemia of the spinal cord that leads to increased production of circulating factors such as adenosine or bradykinin. It has, however, been shown to induce local adenosine release. $^{32}$ Therefore, the possibility cannot be discounted that intrathecal opioids may trigger the 


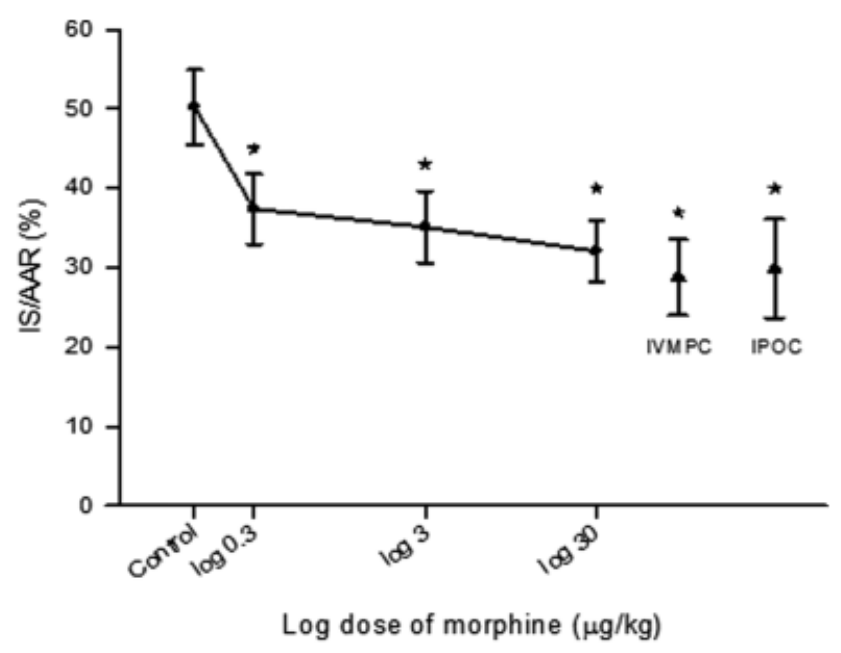

Fig. 2. Curve illustrating the relationship between IS/ARR and dose of intrathecal morphine. This is compared with intravenous morphine and ischemic post-conditioning. IS/AAR, infarct size as percentage of area at risk; IVMPC, intravenous post-conditioning; $I P o C$, ischemic post-conditioning.

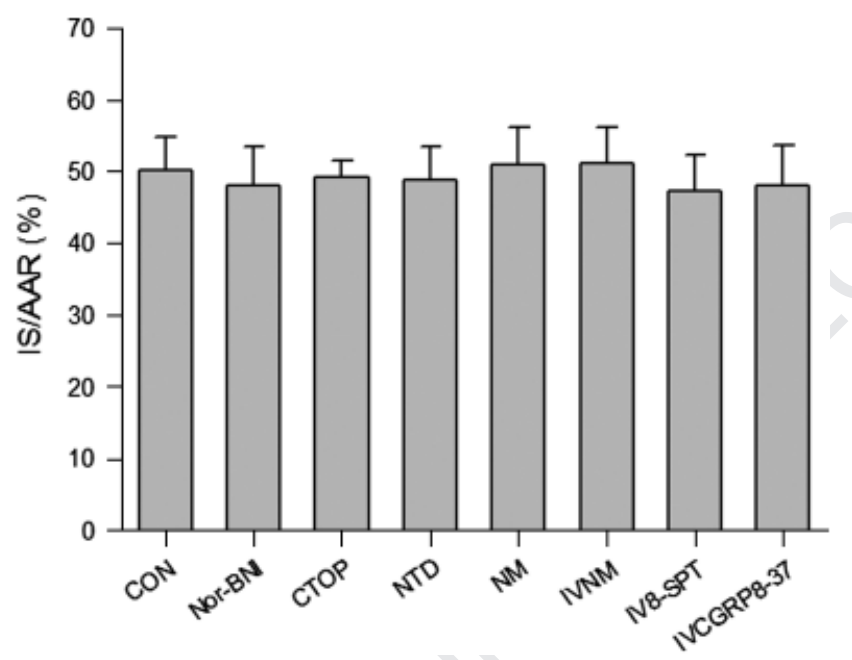

Fig.3. The effect on IS/AAR by opioid, adenosine or CGRP receptor antagonist. Data expressed as percentage $\pm S D$. No difference was detected between any of the group compared with control. ANOVA was used for analysis. IS/AAR, infarct size as percentage of area at risk; $M P C$, morphine post-conditioning at $3 \mu \mathrm{g} / \mathrm{kg}$; Nor-BNI, Nor-binaltorphimin; CTOP, D-Phe-Cys-Tyr-DTrp-Orn-Thr-Pen-Thr-NH2; naltrindole (NTD); NM, naloxone methiodide; IVNM, intravenous naloxone methiodide; 8-SPT, 8- $\rho$ sulfophenyl theophylline and CGRP ${ }_{8-37}$, human fragment 8-37 of calcitonin gene-related peptide.

release of such factors from distal organs or nerve endings. From our results, it is possible that peripheral adenosine receptors are involved as the nonspecific adenosine receptor antagonist 8-SPT does not cross the blood-brain barrier. ${ }^{33}$

Inflammatory activity from neutrophils contribute to the extension of the infarct following

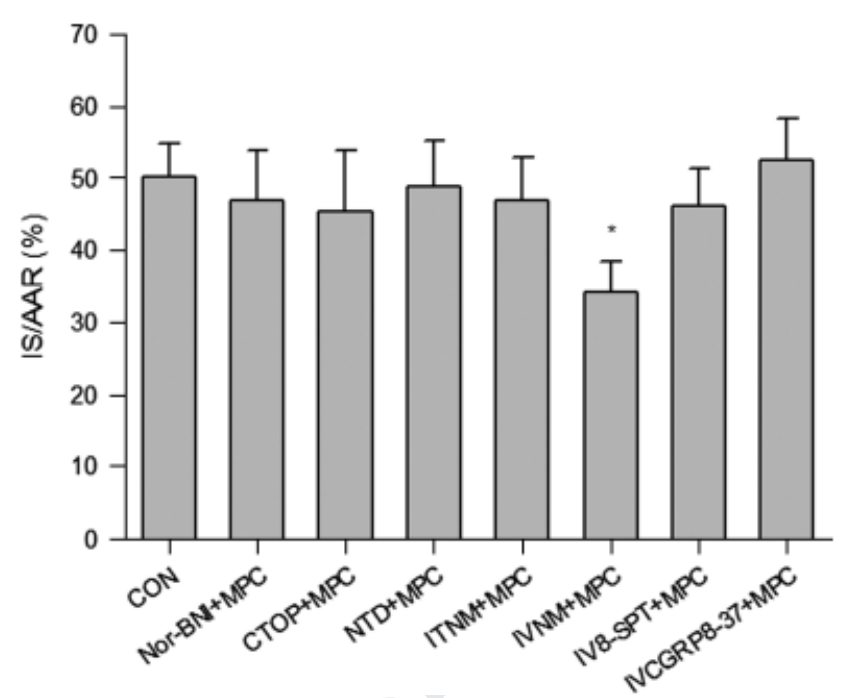

Fig. 4. The effect on IS/AAR by the administration of opioid, adenosine and CGRP receptor antagonists before intrathecal morphine post-conditioning $(3 \mu \mathrm{g} / \mathrm{kg})$. Data expressed as percentage $\pm S D$. ANOVA was used for analysis. IS/AAR, infarct size as percentage of area at risk; MPC, morphine post-conditioning at $3 \mu \mathrm{g} / \mathrm{kg}$; Nor BNI, Nor-binaltorphimine; CTOP, D-Phe-Cys-TyrD-Trp-Orn-Thr-Pen-Thr-NH2; naltrindole (NTD); NM, naloxone methiodide; IVNM, intravenous naloxone methiodide; 8-SPT, 8- $\rho-$ sulfophenyl theophylline and $C G R P_{8-37}$, human fragment 8-37 of calcitonin gene-related peptide. ${ }^{*} \mathrm{P}<0.01$ compared with control.

reperfusion. ${ }^{34}$ Systemic morphine has anti-inflammatory properties ${ }^{35}$ and, when administered as a pre-conditioning agent in rat models of myocardial infarction, it can attenuate neutrophil-endothelium interactions, a necessary component for tissue injury. ${ }^{36}$ Although thought to be peripherally mediated, a central mechanism of the anti-inflammatory effect of morphine has been proposed and segmental action of intrathecal morphine has been shown to inhibit inflammatory paw edema. ${ }^{37} \mathrm{How}^{-}$ ever, in that same study, intrathecal morphine had no effect on myeloperoxidase levels, a marker of neutrophil activity, implying a lesser role for neutrophils in its peripheral anti-inflammatory effects. Therefore, if anti-inflammatory effects contribute to infarct sparing with intrathecal morphine postconditioning, it may not necessarily be by altering neutrophil activity.

As ischemic and pharmacological post-conditioning can also be elicited in isolated heart models, ${ }^{13,30,38}$ neural involvement appears to be not absolutely necessary in remote post-conditioning. However, remote pre-conditioning research has demonstrated that the interruption of autonomic transmission can abrogate the effect of protective ischemia. ${ }^{39,40}$ Similar results have not been 
demonstrated for remote post-conditioning. However, CGRP is a neurotransmitter that is released by capsaicin-sensitive sensory nerves and its antagonism abrogates the infarct-sparing effect of intrathecal morphine. This would suggest that an involvement of peripheral CGRP receptors as the antagonist $\mathrm{CGRP}_{8-37}$ is not known to freely penetrate the blood-brain barrier. ${ }^{41}$ Therefore, the results from this study suggest a contribution of the central nervous system as an initiator of myocardial protection. Whether it may participate in other forms of remote pre- or post-conditioning remains to be determined.

Many aspects of remote post-conditioning remain elusive. ${ }^{3,42}$ Like other complex physiological systems, multiple pathways with inbuilt redundancy may exist with the system of innate protection from IR and the central nervous system may play an integral part either in triggering or in transmitting the protective signals. Multimodal treatment of hypertension and pain are well established in clinical practice and work adopting this approach to cardiac protection has demonstrated augmented response for both pre- and postconditioning. ${ }^{4-45}$ This implies that maximal protection may not be achievable by a single approach. A greater understanding of the triggering mechanisms therefore would potentially enable one to harness the maximal protective effects from myocardial conditioning strategies.

\section{Acknowledgements}

Financial support: Departmental funding.

Conflicts of interests: None.

\section{References}

1. Buja LM. Myocardial ischemia and reperfusion injury. Cardiovasc Pathol 2005; 14: 170-5.

2. Monassier JP. Reperfusion injury in acute myocardial infarction. From bench to cath lab. Part I: basic considerations. Archiv Cardiovasc Diseases 2008; 101: 491-500.

3. Granfeldt A, Lefer DJ, Vinten-Johansen J. Protective ischaemia in patients: preconditioning and postconditioning. Cardiovasc Res 2009; 83: 234-46.

4. Gritsopoulos G, Iliodromitis EK, Zoga A, Farmakis D, Demerouti E, Papalois A, Paraskevaidis IA, Kremastinos DT. Remote postconditioning is more potent than classic postconditioning in reducing the infarct size in anesthetized rabbits. Cardiovasc Drugs Ther 2009; 23: 193-8.
5. Zatta AJ, Kin H, Yoshishige D, Jiang R, Wang N, Reeves JG, Mykytenko J, Guyton RA, Zhao Z-Q, Caffrey JL, VintenJohansen J. Evidence that cardioprotection by postconditioning involves preservation of myocardial opioid content and selective opioid receptor activation. Am J Physiol Heart Circ Physiol 2008; 294: H1444-51.

6. Jang Y, Xi J, Wang H, Mueller RA, Norfleet EA, Xu Z. Postconditioning prevents reperfusion injury by activating [delta]-opioid receptors. Anesthesiology 2008; 108: 243-50.

7. Chen Z, Li T, Zhang B. Morphine postconditioning protects against reperfusion injury in the isolated rat hearts. J Surg Res 2008; 145: 287-94.

8. McPherson BC, Yao Z. Morphine mimics preconditioning via free radical signals and mitochondrial KATP, channels in myocytes. Circulation 2001; 103: 290-5.

9. Wong G, Li R, Jiang LL, Irwin M. Remifentanil postconditioning attenuates cardiac ischemia-reperfusion injury via kappa or delta opioid receptor activation. Acta Anaesthesiol Scand 2010; 54: 510-8.

10. Zhang Y, Irwin MG, Wong TM. Remifentanil preconditioning protects against ischemic injury in the intact rat heart. Anesthesiology 2004; 101: 918-23.

11. Li R, Wong GT, Wong TM, Zhang Y, Xia Z, Irwin MG. Intrathecal morphine preconditioning induces cardioprotection via activation of delta, kappa, and mu opioid receptors in rats. Anesth Analg 2009; 108: 23-9.

12. Wong GTC, Ling Ling J, Irwin MG. Activation of central opioid receptors induces cardioprotection against ischemia-reperfusion injury. Anesthesia Analgesia 2009; Epub ahead of print.

13. Yang XM, Phili $\bar{\equiv}$ Downey JM, Cohen MV. Postconditioning's protection is not dependent on circulating blood factors or cells but involves adenosine receptors and requires PI3-kinase and guanylyl cyclase activation. Basic Res Cardiol 2005; 100: 57-63.

14. Maggi CA. Tachykinins and calcitonin gene-related peptide (CGRP) as co-transmitters released from peripheral endings of sensory nerves. Prog Neurobiol 1995; 45: 1-98.

15. Lu R, Hu CP, Deng HW, Li YJ. Calcitonin gene-related peptide-mediated ischemic preconditioning in the rat heart: influence of age. Regul Pept 2001; 99: 183-9.

16. Li D, Li NS, Chen QQ, Guo R, Xu PS, Deng HW, Li YJ. Calcitonin gene-related peptide-mediated cardioprotection of postconditioning in isolated rat hearts. Regul Pept 2008; 147: 4-8.

17. Yaksh TL, Rudy TA. Chronic catheterization of the spinal subarachnoid space. Physiol Behav 1976; 17: 1031-6.

18. Liu GS, Thornton J, Van Winkle DM, Stanley AW, Olsson RA, Downey JM. Protection against infarction afforded by preconditioning is mediated by A1 adenosine receptors in rabbit heart. Circulation 1991; 84: 350-6.

19. Wolfrum S, Nienstedt J, Heidbreder M, Schneider K, Dominiak P, Dendorfer A. Calcitonin gene related peptide mediates cardioprotection by remote preconditioning. Regul Pept 2005; 127: 217-24.

20. Groenendaal D, Freijer J, de Mik D, Bouw MR, Danhof M, de Lange EC. Influence of biophase distribution and Pglycoprotein interaction on pharmacokinetic-pharmacodynamic modelling of the effects of morphine on the EEG. Br J Pharmacol 2007; 151: 713-20.

21. Gross ER, Hsu AK, Gross GJ. Acute aspirin treatment abolishes, whereas acute ibuprofen treatment enhances morphine-induced cardioprotection: role of 12-lipoxygenase. J Pharmacol Exp Ther 2004; 310: 185-91.

22. Kerendi F, Kin H, Halkos ME, Jiang R, Zatta AJ, Zhao ZQ, Guyton RA, Vinten-Johansen J. Remote postconditioning. 


\section{J. Ling Ling et al.}

Brief renal ischemia and reperfusion applied before coronary artery reperfusion reduces myocardial infarct size via endogenous activation of adenosine receptors. Basic Res Cardiol 2005; 100: 404-12.

23. Chiari PC, Bienengraeber MW, Pagel PS, Krolikowski JG, Kersten JR, Warltier DC. Isoflurane protects against myocardial infarction during early reperfusion by activation of phosphatidylinositol-3-kinase signal transduction: evidence for anesthetic-induced postconditioning in rabbits. Anesthesiology 2005; 102: 102-9.

24. Feng J, Lucchinetti E, Ahuja P, Pasch T, Perriard JC, Zaugg M. Isoflurane postconditioning prevents opening of the mitochondrial permeability transition pore through inhibition of glycogen synthase kinase $3 \beta$. Anesthesiology 2005; 103: 987-95.

25. Jin ZX, Zhou JJ, Xin M, Peng DR, Wang XM, Bi SH, Wei XF, Yi DH. Postconditioning the human heart with adenosine in heart valve replacement surgery. Ann Thorac Surg 2007; 83: 2066-72.

26. Penna C, Mancardi D, Rastaldo R, Losano G, Pagliaro P. Intermittent activation of bradykinin B2 receptors and mitochondrial KATP channels trigger cardiac postconditioning through redox signaling. Cardiovasc Res 2007; 75: 168-77.

27. Mudalagiri NR, Mocanu MM, Di Salvo C, Kolvekar S, Hayward M, Yap J, Keogh B, Yellon DM. Erythropoietin protects the human myocardium against hypoxia/reoxygenation injury via phosphatidylinositol-3 kinase and ERK1/2 activation. Br J Pharmacol 2008; 153: 50-6.

28. Hausenloy DJ, Yellon DM. Preconditioning and postconditioning: united at reperfusion. Pharmacol Therap 2007; 116: 173-91.

29. Hausenloy DJ, Yellon DM. Preconditioning and postconditioning: underlying mechanisms and clinical application. Atherosclerosis 2009; 204: 334-41.

30. Shimizu M, Tropak M, Diaz RJ, Suto F, Surendra H, Kuzmin E, Li J, Gross G, Wilson GJ, Callahan J, Redington AN. Transient limb ischaemia remotely preconditions through a humoral mechanism acting directly on the myocardium: evidence suggesting cross-species protection. Clin Sci (Lond) 2009; 117: 191-200.

31. Loukogeorgakis SP, Williams R, Panagiotidou AT, Kolvekar SK, Donald A, Cole TJ, Yellon DM, Deanfield JE, MacAllister RJ. Transient limb ischemia induces remote preconditioning and remote postconditioning in humans by a KATP channeldependent mechanism. Circulation 2007; 116: 1386-95.

32. Eisenach JC, Hood DD, Curry R, Sawynok J, Yaksh TL, Li X. Intrathecal but not intravenous opioids release adenosine from the spinal cord. J Pain 2004; 5: 64-8.

33. Evoniuk G, von Borstel RW, Wurtman RJ. Antagonism of the cardiovascular effects of adenosine by caffeine or 8-(p-sulfophenyl)theophylline. J Pharmacol Exp Ther 1987; 240: 428-32.

34. Vinten-Johansen J. Involvement of neutrophils in the pathogenesis of lethal myocardial reperfusion injury. Cardiovasc Res 2004; 61: 481-97.
35. Planas E, Sanchez S, Rodriguez L, Pol O, Puig MM. Antinociceptive/anti-edema effects of liposomal morphine during acute inflammation of the rat paw. Pharmacology 2000; 60: 121-7.

36. Wang T-L, Chang H, Hung C-R, Tseng Y-Z. Morphine preconditioning attenuates neutrophil activation in rat models of myocardial infarction. Cardiovasc Res 1998; 40: 557-63.

37. Brock SC, Tonussi CR. Intrathecally injected morphine inhibits inflammatory paw edema: the involvement of nitric oxide and cyclic-guanosine monophosphate. Anesth Analg 2008; 106: 965-71.

38. Wong GT, Li R, Jiang LL, Irwin MG. Remifentanil postconditioning attenuates cardiac ischemia-reperfusion injury via kappa or delta opioid receptor activation. Acta Anaesthesiol Scand 2009; in press.

39. Gho BCG, Schoemaker RG, Van den Doel MA, Duncker DJ, Verdouw PD. Myocardial protection by brief ischemia in noncardiac tissue. Circulation 1996; 94: 2193-200.

40. Loukogeorgakis SP, Panagiotidou AT, Broadhead MW $\bar{\equiv}$ ald A, Deanfield JE, MacAllister RJ. Remote ischemic preconditioning provides early and late protection against endothelial ischemia-reperfusion injury in humans: role of the autonomic nervous system. J Am Coll Cardiol 2005; 46: 450-6.

41. Edvinsson L, Nilsson E, Jansen-Olesen I. Inhibitory effect of BIBN4096BS, CGRP(8-37), a CGRP antibody and an RNASpiegelmer on CGRP induced vasodilatation in the perfused and non-perfused rat middle cerebral artery. Br J Pharmacol 2007; 150: 633-40.

42. Dow J, Bhandari A, Kloner RA. The mechanism by which ischemic postconditioning reduces reperfusion arrhythmias in rats remains elusive. J Cardiovasc Pharmacol Ther 2009; 14: 99-103.

43. Mullenheim J, Ebel D, Bauer M, Otto F, Heinen A, Frassdorf J, Preckel B, Schlack W. Sevoflurane confers additional cardioprotection after ischemic late preconditioning in rabbits. Anesthesiology 2003; 99: 624-31.

44. Rosanio S, Ye Y, Atar S, Rahman AM, Freeberg SY, Huang $\mathrm{MH}$, Uretsky BF, Birnbaum Y. Enhanced cardioprotection against ischemia-reperfusion injury with combining sildenafil with low-dose atorvastatin. Cardiovasc Drugs Ther 2006; 20: 27-36.

45. Weihrauch D, Krolikowski JG, Bienengraeber M, Kersten JR, Warltier DC, Pagel PS. Morphine enhances isofluraneinduced postconditioning against myocardial infarction: the role of phosphatidylinositol-3-kinase and opioid receptors in rabbits. Anesth Analg 2005; 101: 942-9.

Address:

Dr Gordon Wong

Room 424, K Block

Queen Mary Hospital

Pokfulam Road

Hong Kong

e-mail: gordon@hku.hk 